\title{
Preretinal neovascularisation associated with choroidal melanoma
} Jennifer Lee, Sanjay Logani, Hesham Lakosha, Robert P Schroeder, Rand Simpson,
Lee M Jampol

\begin{abstract}
Background-The rare occurrence of iris neovascularisation and choroidal (subretinal) neovascularisation in patients with choroidal melanoma has been reported. However, the occurrence of preretinal neovascularisation (NVE) fed from the retinal circulation in eyes with choroidal melanoma is far less frequently reported.

Methods-Three case reports of choroidal melanoma with the very rare finding of overlying NVE.

Results-The three patients had choroidal melanomas, localised serous retinal detachment, and NVE. Two cases showed definite retinal capillary non-perfusion, and one of these two cases demonstrated retinal telangiectasis. One patient's melanoma responded quickly to iodine125 plaque radiotherapy; however, the retinal neovascularisation persisted and caused vitreous haemorrhage. Localised scatter photocoagulation was successful in causing the complete regression of the neovascularisation. The other two patients had their eyes enucleated (one with planned pre-enucleation external beam radiotherapy). Demonstration of preretinal vessels in one of the cases was possible in histological sections.

Conclusion-Preretinal neovascularisation may occur as a complication of choroidal melanoma. Possible aetiologies include the release of tumour angiogenic factors, inflammation, chronic retinal detachment with secondary retinal ischaemia, retinal vascular occlusion secondary to retinal vessel invasion by the tumour, or following radiation therapy. Optimal management of the neovascularisation is not known at this time. Supplemental localised scatter photocoagulation may be of benefit in some cases.

(Br f Ophthalmol 2001;85:1309-1312)
\end{abstract}

Preretinal neovascularisation (NVE) fed from the retinal circulation is commonly associated with ischaemic retinal vascular diseases and is thought to be due to production of angiogenic growth factors induced by the retinal ischaemia (and probably hypoxia). Choroidal melanoma has previously been associated with neovascularisation of the iris (large tumours, total retinal detachment, or following retinal vascular occlusions or radiation). ${ }^{1}$ Subretinal neovascularisation fed from the choroid has been described by Lubin et al.$^{2}$ Neovascularisation of the retina or disc is apparently very rare: Vine $\mathrm{e}^{3}$ and Johnson et $a l^{4}$ have reported a total of two cases of choroidal melanoma associated with retinal neovascularisation. We present three patients with choroidal melanoma with associated NVE and discuss the mechanism of development of the neovascularisation and the response to therapy in the one case not enucleated.

\section{Case reports}

CASE 1

A 35 year old white woman complained of flashing lights and blurred vision inferiorly for 2 days. Best corrected visual acuity was right eye $6 / 5$ and left eye $6 / 7.5$. There was a left afferent pupillary defect. Applanation tonometry and slit lamp biomicroscopy were normal. The right fundus was normal. Ophthalmoscopy revealed a medium sized, amelanotic, left choroidal mass under the inferior arcade (Fig 1). The tumour measured $7.5 \mathrm{~mm}$ $\times 8.5 \mathrm{~mm} \times 4.2 \mathrm{~mm}$. There was an overlying serous retinal detachment that extended to the fovea and retinal pigment epithelial changes overlying the tumour. There was beading of an overlying retinal vein and an area of retinal non-perfusion with adjacent NVE just inferior to the tumour in the area of the serous retinal detachment. Marked telangiectasis of the vascular bed was present. The retinal arteries were attenuated.

Fluorescein angiography of the left eye showed abnormal retinal vasculature overlying the tumour with dilatation and telangiectasia. Ischaemia was present and there were fronds of retinal neovascularisation (Fig 2). In the late phase, there was fluorescein leakage from the tumour vessels and retinal neovascular networks. Ultrasonography of the left eye revealed low to medium internal reflectivity on A scan

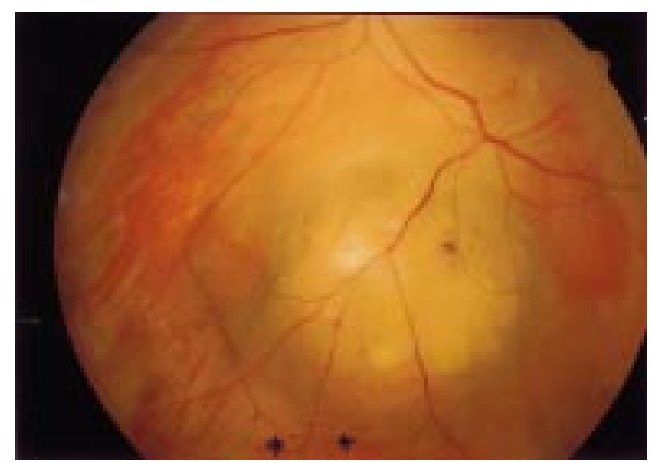

Figure 1 Case 1. A medium sized, amelanotic, choroidal melanoma is seen with overlying serous retinal detachment that extends to the fovea. There are retinal pigment epithelial changes overlying the tumour. There is beading of overlying retinal veins and small fronds of retinal neovascularisation just inferior to the tumour. 


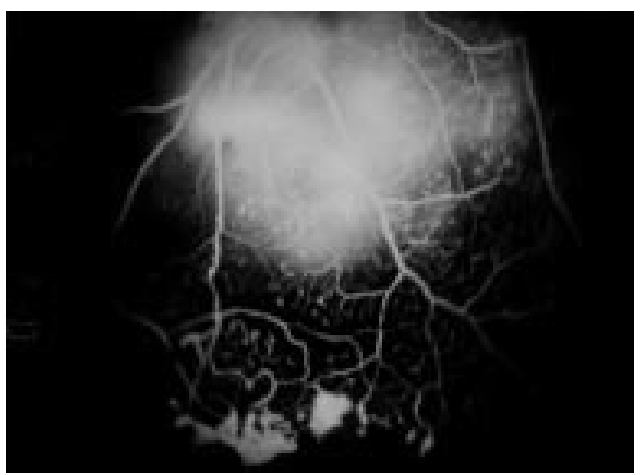

Figure 2 Case 1. Fluorescein angiogram reveals abnormal retinal vasculature in the area of the tumour with dilatation and telangiectasia. Inferior to the tumour, the retinal vasculature ends abruptly and there are fronds of retinal neovascularisation. There is non-perfusion peripheral to the neovascularisation.

and a solid choroidal mass without extrascleral extension on B scan. Systemic evaluation was normal. The patient's tumour was treated with an iodine-125 radioactive episcleral plaque. The tumour was still present after 17 days of follow up after removal of the iodine-125 radioactive episcleral plaque; however, it had evolved into a flat scar by the subsequent follow up visit at 2 months. The retinal neovascularisation, however, persisted and caused vitreous haemorrhage 3 months after radiation therapy, which was subsequently treated with scatter photocoagulation in the area of NVE (argon green, No 210, 0.1 seconds, 200-300 $\mathrm{mW}$ ). The NVE promptly regressed. The patient is doing well 7 years later with visual acuity 6/6 left eye, no neovascularisation present, a flat scar where the tumour was located, and slight radiation retinopathy (Fig 3). There has been no local or distant tumour recurrence.

CASE 2

A 31 year old white man complained of a superior field defect and floaters for 1 month. Best corrected visual acuity was right eye $6 / 5$ and left eye 6/6. There was no afferent pupillary defect. Slit lamp biomicroscopy was normal. The right fundus was normal. Examination of the left eye revealed an elevated subretinal mass at the 6 o'clock position. The tumour measured $16.5 \mathrm{~mm} \times 14 \mathrm{~mm} \times 5.9 \mathrm{~mm}$. The lesion showed a mixed pigmentation with retinal haemorrhages on its surface. There was an area of subretinal fluid nasal to the lesion. Tufts

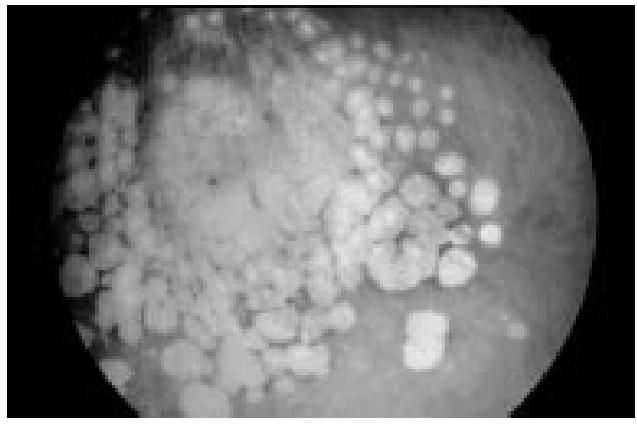

Figure 3 Case 1. Following brachytherapy and scatter photocoagulation, the tumour and neovascularisation have regressed.

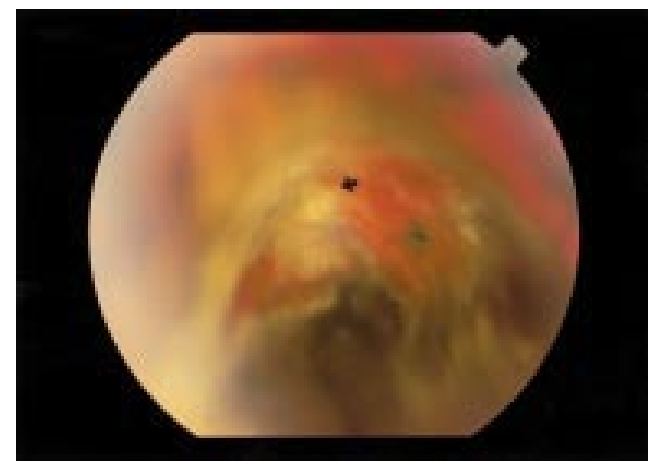

Figure 4 Case 2. Colour fundus photograph demonstrates elevated choroidal mass with haemorrhage and fine preretinal vessels visible (arrow).

of NVE were observed over the surface of the tumour (Fig 4).

Fluorescein angiography (Fig 5A and B) demonstrated a network of preretinal blood vessels that progressively leaked. There was blocked fluorescence as a result of the presence of retinal blood and pigment and hyperfluorescence of part of the tumour periphery, due to atrophy of the overlying retinal pigment epithelium. There was no evidence of a tumour circulation. Ultrasonography of the left eye revealed somewhat variable internal reflectivity. A collar button mass lesion emerged from the choroid, and it had an area of subretinal fluid around it. Ultrasound biomicroscopy showed no evidence of ciliary body invasion. Complete physical examination and laboratory tests were normal. The eye underwent enucleation.

The enucleated globe contained an irregularly shaped tumour in the anterior choroid, abutting the ora serrata. It comprised a mixture of spindle B and a large number of epithelioid cells. Twelve mitotic figures were identified per 40 high power fields. Focally, there were occasional lymphoid follicles. There was an associated serous retinal detachment and marked degenerative changes of overlying retina. Bruch's membrane was penetrated by the tumour with focal invasion of the retina. Preretinal neovascularisation was evident in the vicinity of the tumour (Fig 6). No retinal vascular occlusion or tumour invasion of retinal vessels was seen. No extraocular extension was present.

\section{CASE 3}

A 46 year old white woman complained of blurred and distorted vision in the right eye for 3 months. Best corrected visual acuity was right eye $6 / 60$ and left eye $6 / 6$. There was a right afferent pupillary defect. Applanation tonometry was normal. Slit lamp biomicroscopy was only significant for pigment cells in the right anterior vitreous. Fundus examination of the right eye revealed a large, amelanotic, choroidal mass in the inferotemporal periphery. The tumour measured $17.5 \mathrm{~mm} \times$ $15.0 \mathrm{~mm} \times 8.6 \mathrm{~mm}$. There was a large overlying serous retinal detachment that extended past the macula and superiorly. There were several small areas of NVE on the surface of 

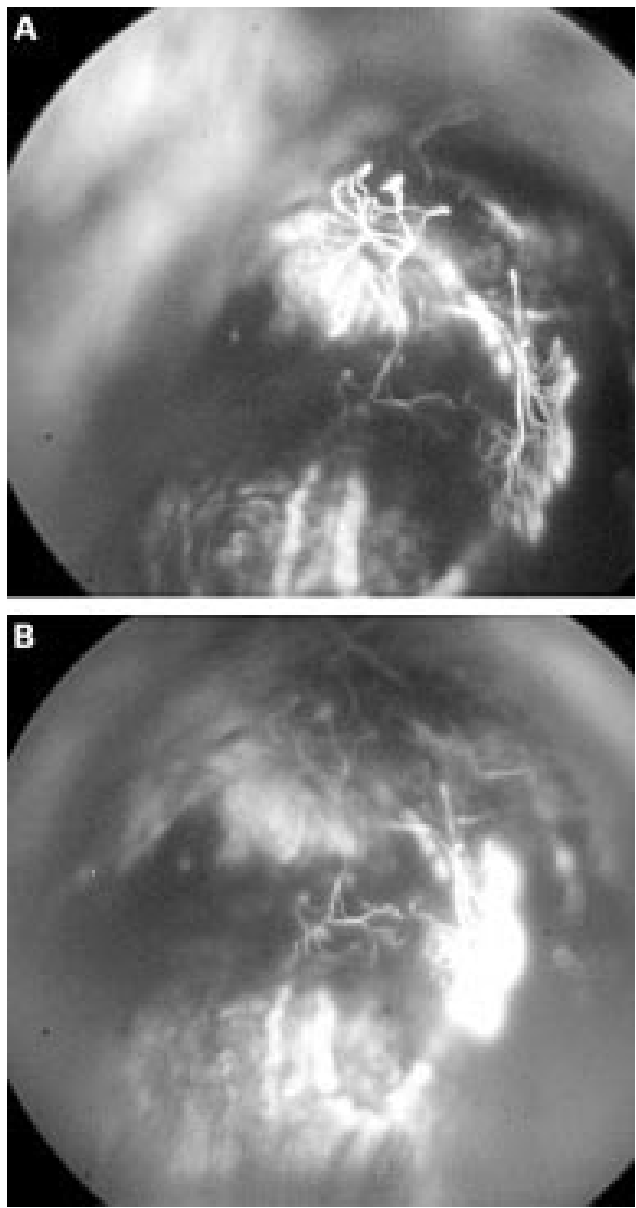

Figure 5 (A) Case 2. Fluorescein angiogram demonstrates fronds of preretinal vessels. (B) Case 2. Late phase of the angiogram shows leakage from the new vessels.

the tumour (Fig 7). The fundus examination of the left eye was normal.

Fluorescein angiography of the right eye showed blockage by pigment and abnormal vessels on the surface of the tumour that leaked fluorescein (Fig 8). Ultrasonography of the right eye revealed low to medium internal reflectivity on $\mathrm{A}$ scan and a solid choroidal mass without extrascleral extension on B scan.

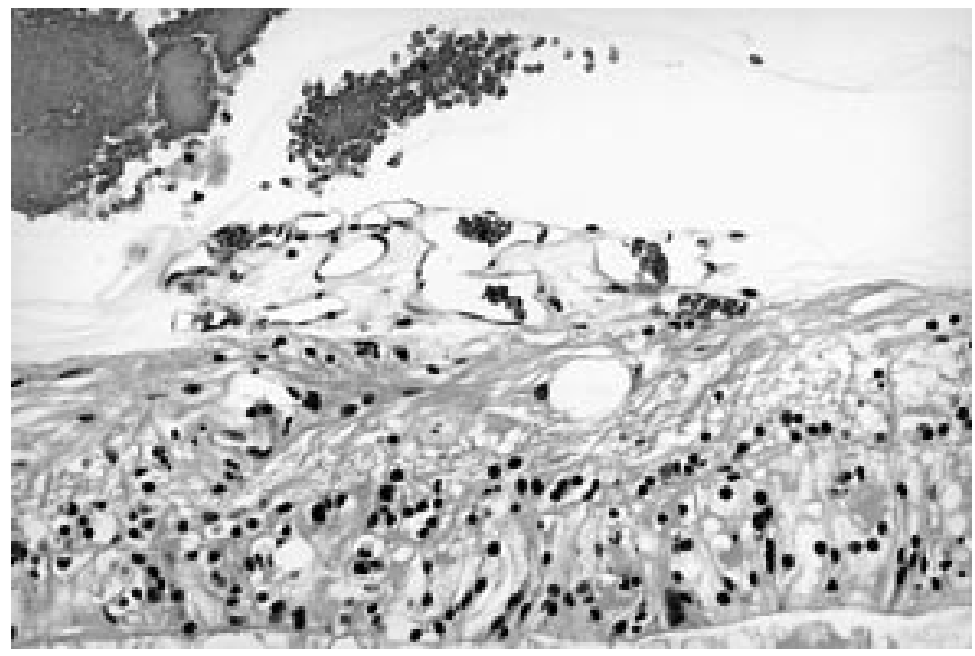

Figure 6 Case 2. Photomicrograph clearly shows preretinal neovascularisation. The underlying retina is degenerated and disorganised. The retina is detached from the underlying tumour. (Haematoxylin and eosin, original magnification $\times 40$ ).

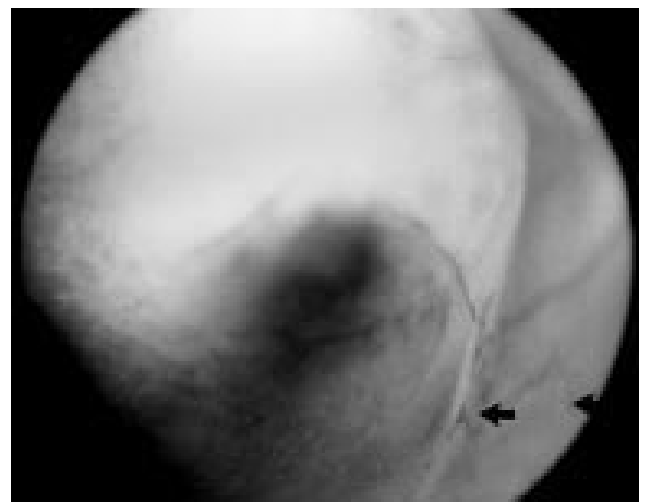

Figure 7 Case 3. A large, amelanotic, choroidal melanoma is seen with surrounding serous retinal detachment that extended into the macula. Retinal neovascularisation was present at the inferonasal edge of the tumour (arrows).

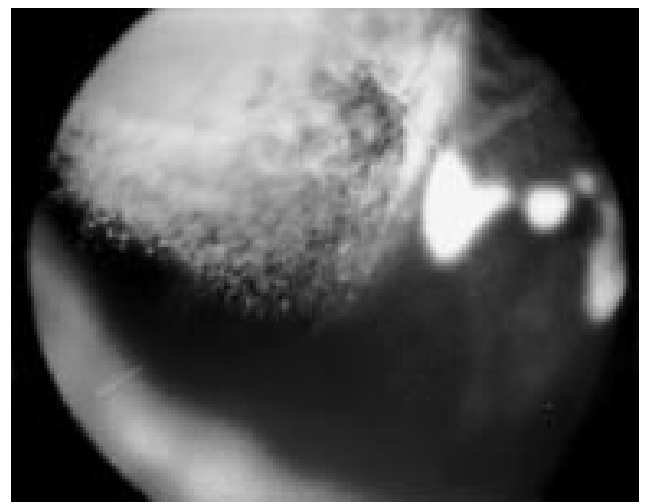

Figure 8 Case 3. Fluorescein angiogram reveals leakage of neovascular fronds. The angiogram suggests adjacent non-perfusion.

Complete physical examination and laboratory tests were normal. The patient's right eye received planned pre-enucleation external beam radiation and was subsequently enucleated. We were unable to identify retinal neovascularisation on the limited sections available. The patient is doing well without local or distant recurrence with 5 years of follow up.

\section{Discussion}

Iris neovascularisation has been associated with large choroidal melanomas, especially in the presence of exudative retinal detachments. ${ }^{1}$ It may also be seen with necrotic melanomas, retinal vascular occlusions, or with radiation treatment of large tumours. ${ }^{1}$ Although usually considered evidence in favour of the benign nature of an elevated, pigmented choroidal lesion, choroidal (subretinal) neovascularisation has been reported in association with choroidal melanoma. ${ }^{25}$ The association of preretinal neovascularisation (NVE) with choroidal melanoma is very rare. To our knowledge there are only two other reported cases of NVE associated with choroidal melanoma. ${ }^{34}$ With our three patients there are now five known cases. Our cases 2 and 3, as well as both of those reported previously in the literature, were large sized melanomas. All five cases had an associated serous retinal detachment. None of 
the patients had received radiation before the discovery of the neovascularisation.

The possible mechanisms of retinal neovascularisation associated with choroidal melanoma include: (1) production of tumour angiogenic factor, ${ }^{4-8}$ (2) serous retinal detachment with secondary retinal ischaemia, (3) retinal vascular occlusion secondary to retinal invasion by the tumour, (4) inflammation, (5) radiation retinopathy after treatment. It is unclear if choroidal tumours can produce enough angiogenic factors to affect the overlying retinal vasculature and produce capillary telangiectasis and neovascularisation. Secondary retinal ischaemia from serous retinal detachment is a plausible cause for retinal neovascularisation associated with choroidal melanoma. All five cases reported to date had an associated serous retinal detachment. Serous retinal elevation separates the outer retina from the nutritive choroidal circulation and may impair retinal blood flow as well. Two of our cases (cases 1 and 3) showed definite capillary non-perfusion indicative of retinal ischaemia. The telangiectasis and NVE in case 1 may have developed secondary to the release of angiogenic factors by the ischaemic retina.

Choroidal melanoma can also cause retinal artery and vein occlusion or uveitis. Retinal or disc neovascularisation can be secondary to retinal vaso-occlusive disease. ${ }^{9}$ In none of the five cases reported to date (including our cases) were any obvious retinal vascular occlusions seen. Inflammation induced by the tumour may also play a part in the development of neovascularisation.

It is not currently known what is the best management of preretinal neovascularisation from choroidal melanoma. Alternatives for treatment of the tumour include episcleral plaque radiotherapy, charged particle radiotherapy, thermotherapy, or enucleation. Case 1 had radioactive plaque therapy with a rapid reduction in the tumour to a flat scar. The area of NVE, however, persisted and caused vitreous haemorrhage. The neovascularisation was treated with local scatter photocoagulation and responded well. In theory, since retinal vessels are somewhat radiosensitive, retinal neovascularisation might regress with radioactive plaque therapy; however, this was not seen in our patient. Supplemental localised scatter photocoagulation would seem reasonable therapy for preretinal neovascularisation from choroidal vasculature when the NVE causes vitreous haemorrhage, especially when the tumour has regressed after therapy such as radiation (as in case 1), or transpupillary thermotherapy.

The authors have no proprietary interest in this article.

This research was supported in part by an unrestricted grant from Research to Prevent Blindness, Inc, New York City (Northwestern University).

S Logani was Heed Ophthalmic Foundation Heed/Knapp fellow for $1995-6$.

1 Gartner S, Henkind P. Neovascularization of the iris (rubeosis iridis). Surv Ophthalmol 1978;22:291-312.

2 Lubin JR, Gragoudas ES, Albert DM. Choroidal neovascularization associated with malignant melanoma: a case larization associated with malignant mel
report. Acta Ophthalmol 1982;60:412-18.

3 Vine AK. Choroidal melanoma with disc and retinal neovascularization. Retina 1983;3:118-20.

4 Johnson RN, Irvine AR, Char DH. Inferences from beading of a retinal vein draining a choroidal melanoma. $\mathrm{Br} \mathcal{F} \mathrm{Oph}-$ thalmol 1986;70:764-5.

5 Gass J D M. Pathogenesis of disciform detachment of the neuroepithelium VI. Disciform detachment secondary to heredodegenerative, neoplastic, and traumatic lesions of the choroid. Am f Ophthalmol 1967;63:573-611.

6 Folkman J, Merler E, Abernathy C, et al. Isolation of a tumor folkman J, Merler E, Abernathy C, et al. Isolation of a tumor factor respo

7 Cavallo T, Sade R, Folkman J, et al. Tumor angiogenesis: rapid induction of endothelial mitoses demonstrated by autoradiography. F Cell Biol 1972;54:408-20.

8 Finkelstein D, Brem S, Patz A, et al. Experimental retinal neovascularization induced by intravitreal tumors. $A m$ f Ophthalmol 1977;83:660-4.

9 Jampol LM, Ebroon DA, Goldbaum MH. Peripheral proliferative retinopathies: an update on angiogenesis, etiologies and management. Surv Ophthalmol 1994;38:519-40. 